\title{
Spin-wave propagation in cubic anisotropic materials
}

\author{
Koji Sekiguchi ${ }^{1,2,8}$, Seo-Won Lee ${ }^{3,8}$, Hiroaki Sukegawa ${ }^{4}$, Nana Sato ${ }^{1}$, Se-Hyeok $\mathrm{Oh}^{5}$, Robert D McMichael ${ }^{6}$ \\ and Kyung-Jin Lee ${ }^{3,5,7}$
}

The information carrier of modern technologies is the electron charge, whose transport inevitably generates Joule heating. Spin waves, the collective precessional motion of electron spins, do not involve moving charges and thus avoid Joule heating. In this respect, magnonic devices in which the information is carried by spin waves attract interest for low-power computing. However, implementation of magnonic devices for practical use suffers from a low spin-wave signal and on/off ratio. Here, we demonstrate that cubic anisotropic materials can enhance spin-wave signals by improving spin-wave amplitude as well as group velocity and attenuation length. Furthermore, cubic anisotropic materials show an enhanced on/off ratio through a laterally localized edge mode, which closely mimics the gate-controlled conducting channel in traditional field-effect transistors. These attractive features of cubic anisotropic materials will invigorate magnonics research towards wave-based functional devices. NPG Asia Materials (2017) 9, e392; doi:10.1038/am.2017.87; published online 30 June 2017

\section{INTRODUCTION}

Magnonics is a research field that aims to control and manipulate spin waves in magnetic materials for information processing. ${ }^{1-3}$ Spin waves enable Boolean and non-Boolean computing with low-power consumption. ${ }^{4-10}$ Their wave properties also allow distinct functionalities, ${ }^{11-14}$ such as multi-input/output (nonlinear) operations. ${ }^{15,16}$ Despite significant progress, however, the low signal and on/off ratio of spin waves have been major obstacles to the implementation of magnonic devices for practical use. These obstacles are caused by poor excitation efficiency and propagation losses of spin waves. In this work, we show that cubic anisotropic materials offer more efficient spin-wave propagation than conventional materials.

Our strategy to improve the spin-wave signal is through modification of the dispersion relation. When compared with other waves in solid states, the spin-wave dispersion is highly anisotropic, caused by the long-range magnetostatic interaction. An established way to control the anisotropy of dispersion is to change the relative orientation between the equilibrium magnetization direction $\mathbf{m}$ and the direction of wave vector $\mathbf{k}$ (i.e., backward volume $(\mathbf{m} \| \mathbf{k})$, surface ( $\mathbf{m} \perp \mathbf{k}$ for in-plane $\mathbf{m})$ and forward volume modes $(\mathbf{m} \perp \mathbf{k}$ for out-ofplane $\mathbf{m})$ ). As the dispersion determines all spin-wave properties, proper modification of the dispersion may allow us to improve spin-wave properties. We note that for improved functionalities of spin-wave devices, not only the spin-wave amplitude but also the spin-wave attenuation length and group velocity should be improved.

Here, we introduce an epitaxial Fe film as a waveguide for this purpose. The cubic crystalline anisotropy of epitaxial Fe film provides an additional knob to modify the dispersion by changing the relative orientation between the magnetic easy (or hard) axis and the direction of wave vector $\mathbf{k}$. As we show below, proper tuning of this relative orientation allows enhancement of the spin-wave amplitude by a factor of 28 , as well as of the spin-wave attenuation length and group velocity by several factors. We also show that the cubic anisotropy provides a giant lateral spin-wave asymmetry because of a laterally localized edge mode at zero external field, which may enable three-terminal non-volatile spin-wave logic gates.

\section{MATERIALS AND THEORETICAL FRAMEWORK}

The spin-wave dispersion in cubic anisotropic materials was first derived by Kalinikos et al. ${ }^{17}$ Instead of starting with this known dispersion directly, we describe the problem in a rather general way to gain an insight into how to improve spin-wave properties. We first describe two key requirements in the dispersion relation for improved spin-wave properties. From the spin-wave theory for an in-plane magnetization $\mathbf{m}$ with an assumption that $\mathbf{m}$ varies only along the spin-wave propagation direction, ${ }^{18-21}$ the spin-wave amplitude $A_{S W}$, the group velocity $v_{\mathrm{g}}$ and the attenuation length $\Lambda$ are, respectively, given as (see Supplementary Note 1 for details)

$$
\begin{aligned}
& A_{\mathrm{SW}} \propto \sqrt{\frac{H_{2}}{H_{1}}} \frac{M_{\mathrm{S}}}{\alpha_{\mathrm{G}}\left(H_{1}+H_{2}\right)}, \\
& v_{\mathrm{g}}=\frac{\gamma_{\mathrm{g}} \mu_{0} M_{\mathrm{S}}}{2} \frac{\partial P_{k}}{\partial k}\left(\sqrt{\frac{H_{2}}{H_{1}}} \sin ^{2} \phi-\sqrt{\frac{H_{1}}{H_{2}}}\right), \\
& \Lambda=\frac{M_{\mathrm{S}}}{\alpha_{\mathrm{G}}\left(H_{1}+H_{2}\right)} \frac{\partial P_{k}}{\partial k}\left(\sqrt{\frac{H_{2}}{H_{1}}} \sin ^{2} \phi-\sqrt{\frac{H_{1}}{H_{2}}}\right),
\end{aligned}
$$

\footnotetext{
${ }^{1}$ Department of Physics, Keio University, Yokohama, Japan; ${ }^{2}$ JST-PRESTO, Tokyo, Japan; ${ }^{3}$ Department of Materials Science and Engineering, Korea University, Seoul, Korea; ${ }^{4}$ National Institute for Materials Science (NIMS), Tsukuba, Japan; ${ }^{5}$ Department of Nano-Semiconductor and Engineering, Korea University, Seoul, Korea; ${ }^{6}$ Center for Nanoscale Science and Technology, National Institute of Standards and Technology, Gaithersburg, MD, USA and ${ }^{7}$ KU-KIST Graduate School of Converging Science and Technology, Korea University, Seoul, Korea

8These authors contributed equally to this work.

Correspondence: Professor K-J Lee, Department of Materials Science and Engineering, Korea University, Anam-ro 145, Seongbuk-gu, Seoul 02841, Korea.

E-mail: kj_lee@korea.ac.kr

Received 2 January 2017; revised 7 March 2017; accepted 24 March 2017
} 
where $H_{1}\left(=F_{\phi \phi} / \mu_{0} M_{\mathrm{S}}+M_{\mathrm{S}} P_{k} \sin ^{2} \phi\right)$ and $H_{2}\left(=F_{\theta \theta} / \mu_{0} M_{\mathrm{S}}+M_{\mathrm{S}}\left(1-P_{k}\right)\right)$ are the in-plane and normal effective fields, respectively, and $F$ is the free magnetic energy density without magnetostatic interactions, which are treated separately. The subscripts (i.e., $\theta$ and $\phi$ are the polar and azimuthal angles of $\mathbf{m}$, respectively) on $F$ refer to partial derivatives around equilibrium positions, $P_{k}=1-\left(1-\mathrm{e}^{-|k| d}\right) /|k| d$, where $k$ is the wavenumber, $d$ is the film thickness, $M_{\mathrm{S}}$ is the saturation magnetization, $\alpha_{\mathrm{G}}$ is the Gilbert damping and $\gamma_{\mathrm{g}}$ is the gyromagnetic ratio. In Equation (1), we refer to the dominant in-plane component only because the normal component is negligible due to strong demagnetization of thin film geometry.

Focusing on the limit of $k d \ll 1$ in which $P_{k}$ is small, one finds from Equations (1)-(3), that $A_{\mathrm{SW}}, v_{\mathrm{g}}$ and $\Lambda$ are simultaneously maximized when $H_{1} \approx 0$ (equivalently $F_{\phi \phi} \approx 0$ ) and $\phi \approx \pm \pi / 2$ (i.e., surface mode configuration). Therefore, two key requirements for the improved spin-wave properties are vanishingly small in-plane effective field and surface mode. For $k d \ll 1, F$ consists of the Zeeman and anisotropic energies. The contribution from the Zeeman energy to $F_{\phi \phi}$ is simply an external in-plane field $H(>0)$ that should be applied to ensure the surface mode. Thus, the central question is how to obtain a negative in-plane effective field from the anisotropic energy to diminish $F_{\phi \phi}$ in the surface mode.

We next show that spin-wave propagation where the wave vector $\mathbf{k}$ is along the hard-axis direction of cubic anisotropic materials can naturally satisfy these two requirements. We consider two cases, easy-easy (Figure 1a) and hard-hard (Figure 1b) cases, in which the first (second) word corresponds to the direction of $\mathbf{m}$, and the second word corresponds to the direction of wave vector $\mathbf{k}$. A top view of the waveguide for each case is shown in Figure la and b, where the crystallographic orientations are defined for an epitaxial Fe layer with a cubic crystalline anisotropy. ${ }^{22}$ The long axis of the waveguide (i.e., the direction of wave vector $\mathbf{k}$ ) for the easy-easy device is along the easy axis (Fe [100] direction; Figure 1a), whereas that for the hard-hard device is along the hard axis (Fe [110] direction; Figure 1b). Our main focus is the hard-hard case, while the easy-easy case corresponds to conventional spin-wave propagation and will be used as a reference. In both cases, the external field $H$ is applied in the $y$ direction, and wave vector $(\mathbf{k})$ is in the $x$-direction. The cubic anisotropy energy density $E_{\mathrm{an}}$ is given as

$$
E_{\mathrm{an}}=K_{\mathrm{c}}\left(\cos ^{2} \alpha \cos ^{2} \beta+\cos ^{2} \beta \cos ^{2} \gamma+\cos ^{2} \gamma \cos ^{2} \alpha\right),
$$

where $K_{\mathrm{c}}$ is the cubic anisotropy, and $\alpha\left(=\phi-\phi_{\mathrm{K}}\right), \beta\left(=\pi / 2-\phi+\phi_{\mathrm{K}}\right)$ and $\gamma$ $(=\theta=\pi / 2)$ are direction cosines of the magnetization with respect to the easy axes of cubic anisotropy. Here, $\phi_{\mathrm{K}}$ is the angle between the easy axis and $x$-axis and is $0(\pi / 4)$ for the easy-easy (hard-hard) case. On the other hand, the Zeeman energy is $-\mu_{0} M_{\mathrm{S}} H \sin \theta \sin \phi$. With these energy terms, $H_{1}$ for the hard-hard and easy-easy cases are readily calculated as

$$
\begin{aligned}
& H_{1}^{\text {hard }}=H \sin \phi_{\text {eq }}-H_{\mathrm{A}} \cos \left(4 \phi_{\mathrm{eq}}\right)+M_{\mathrm{S}} P_{k} \sin ^{2} \phi_{\mathrm{eq}}, \\
& H_{1}^{\text {easy }}=H+H_{\mathrm{A}}+M_{\mathrm{S}} P_{k},
\end{aligned}
$$

where $H_{\mathrm{A}}\left(=2 K_{\mathrm{c}} / \mu_{0} M_{\mathrm{S}}\right)$ is the cubic anisotropy field, $\phi_{\mathrm{eq}}=\pi / 2$ for $h \geq 1$, $h=H / H_{\mathrm{A}}$, and

$$
\phi_{\mathrm{eq}}=\sin ^{-1}\left(\frac{6^{1 / 3}+\left(9 h+\sqrt{81 h^{2}-6}\right)^{2 / 3}}{6^{2 / 3}\left(9 h+\sqrt{81 h^{2}-6}\right)^{1 / 3}}\right),
$$

for $0 \leq h<1$.

For $h \geq 1, H_{1}^{\text {hard }}$ becomes $H-H_{\mathrm{A}}+M_{\mathrm{S}} P_{k}$. For the hard-hard case, therefore, the cubic anisotropy provides a negative effective field and $H_{1}^{\text {hard }}$ becomes small when $H \approx H_{\mathrm{A}}$ and $\phi_{\mathrm{eq}}=\pi / 2$ because $M_{\mathrm{S}} P_{k}$ is small. Figure 1c shows that this is indeed the case. For the hard-hard case, the frequency $f(=\omega / 2 \pi)$ minimizes at $H \approx H_{\mathrm{A}}$ (Figure 1d) and all of $A_{\mathrm{SW}}, v_{\mathrm{g}}$ and $\Lambda$ are largely enhanced at $H \approx H_{\mathrm{A}}$, in comparison to the easy-easy case (Figure le and f). We note that the improvements of spin-wave properties discussed in this section are consequences of the known dispersion, ${ }^{17}$ but there has been no direct experimental proof for spin-wave waveguides made of cubic anisotropic materials.

\section{RESULTS AND DISCUSSION}

To confirm the theoretical prediction, we measure spin-wave-induced voltages in the time domain for microfabricated devices containing two antennas (i.e., spin-wave excitation and detection antennas (Figure 2a); see Methods) based on the propagating spin-wave spectroscopy. ${ }^{23,24}$ The layer structure of the waveguide is $\mathrm{Cr}(40) / \mathrm{Fe}$ (25)/Mg(0.45)/Mg-Al(1.2)/oxidation (thicknesses in nanometers; see Methods), where the epitaxial Fe layer has cubic crystalline anisotropy. ${ }^{22} \mu_{0} H_{\mathrm{A}}$ of the Fe film is $(66 \pm 2) \mathrm{mT}$, determined by the vibrating sample magnetometer (Supplementary Note 2 ). We fabricate spin-wave devices with varying antenna distances to measure the group velocity and attenuation length.

Figure $2 \mathrm{~b}-\mathrm{d}$ show representative time-domain results at $H \approx H_{\mathrm{A}}$ at various antenna distances. It clearly shows that the spin-wave amplitude is much larger for the hard-hard case than for the easy-easy case. Magnetic field $H$-dependencies of $A_{\mathrm{SW}}, v_{\mathrm{g}}$ and $\Lambda$ are summarized in Figure 3. All the experimental results show enhanced spin-wave properties at $H \approx H_{\mathrm{A}}$, as predicted by the theory. At $H \approx H_{\mathrm{A}}$ and antenna distance of $5 \mu \mathrm{m}$, the induced voltage of the spin-wave packet reaches $3.30 \mathrm{mV}$ for the hard-hard case (Figure 3a), whereas it is $\sim 0.12 \mathrm{mV}$ for the easy-easy case (Figure 3c). Therefore, the spin-wave amplitude $A_{\mathrm{SW}}$ for the hard-hard case is enhanced by a factor of 28 in comparison with the easy-easy case. By analyzing the antenna-distance dependence of amplitudes and arrival times of spin-wave packets, ${ }^{24}$ we deduce the spin-wave attenuation length $\Lambda$ and the spin-wave group velocity $v_{\mathrm{g}}$. At the enhancement condition $\left(\mu_{0} H=66 \mathrm{mT}\right), \Lambda$ for the hard-hard case is $(17.8 \pm 0.5) \mu \mathrm{m}$, whereas $\Lambda$ for the easy-easy case remains $10.0 \pm 4.5 \mu \mathrm{m}$ (Figure 3f). Furthermore, at the enhancement condition, $v_{\mathrm{g}}$ for the hard-hard case is $(23.4 \pm 0.7) \mathrm{km} \mathrm{s}^{-1}$, whereas $v_{\mathrm{g}}$ for the easy-easy case is $(8.9 \pm 0.3) \mathrm{km} \mathrm{s}^{-1}$ (Figure $3 \mathrm{~g}$ ). We note that uncertainties expressed in this paper are 1 s.d. from the fitting parameters.

Therefore, all these results confirm that spin-wave properties are largely improved at the enhancement condition, which is qualitatively consistent with the theoretical predictions. However, for the hard-hard case, we find that there are interesting quantitative differences between the experimental and theoretical results. For instance, the one-dimensional theory (Figure 1) predicts that the ratio of $A_{\mathrm{SW}}$ at $H=H_{\mathrm{A}}$ to $A_{\mathrm{SW}}$ at $H=0$ is $\approx 3.5$, considering spin-wave attenuation at a distance of $5 \mu \mathrm{m}$. This predicted ratio is much smaller than the experimental one $\left(\approx 9 ; A_{\mathrm{SW}}^{H=0} \approx 0.36 \mathrm{mV}\right.$ and $A_{\mathrm{SW}}^{H=H_{\mathrm{A}}} \approx 3.30 \mathrm{mV}$; see Figure 3a).

To understand this discrepancy, we perform two-dimensional micromagnetic simulations for the hard-hard case (Figure 4). When $\mathbf{m}$ is aligned along one of the easy axes (i.e., $H=0$ and $\phi=\pi / 4$; Figure $4 \mathrm{a})$, the spin wave with $-k(+k)$ is localized at the top-left (bottom-right) edge. The exact opposite trend is obtained when $\mathbf{m}$ is aligned along another easy axis (i.e., $H=0$ and $\phi=3 \pi / 4$; Figure $4 \mathrm{~d}$ ). Moreover, this laterally localized spin-wave edge mode is absent when $H=H_{\mathrm{A}}$ (Figure $4 \mathrm{~b}$ ). This edge mode originates from the anisotropic dispersion relation depicted in Figure 4c. It shows a contour plot of the spin-wave dispersion relation of the hard-hard case at $H=0$. The dispersion relation at the excitation frequency $(10.5 \mathrm{GHz})$ is highlighted. One finds that the wavefronts propagate in the direction of $\mathbf{k}$, determined primarily by the antenna geometry, whereas the group velocity, $v_{\mathrm{g}}=\mathrm{d} \omega / \mathrm{d} k$, is perpendicular to the contour, the oblique direction. This anisotropic propagation leads to the edge localization of spin waves, which in turn leads to an additional difference of $A_{\mathrm{SW}}$ between $H=H_{\mathrm{A}}$ and $H=0$, as our experimental setup detects induced voltage integrated over the full width of the waveguide. 

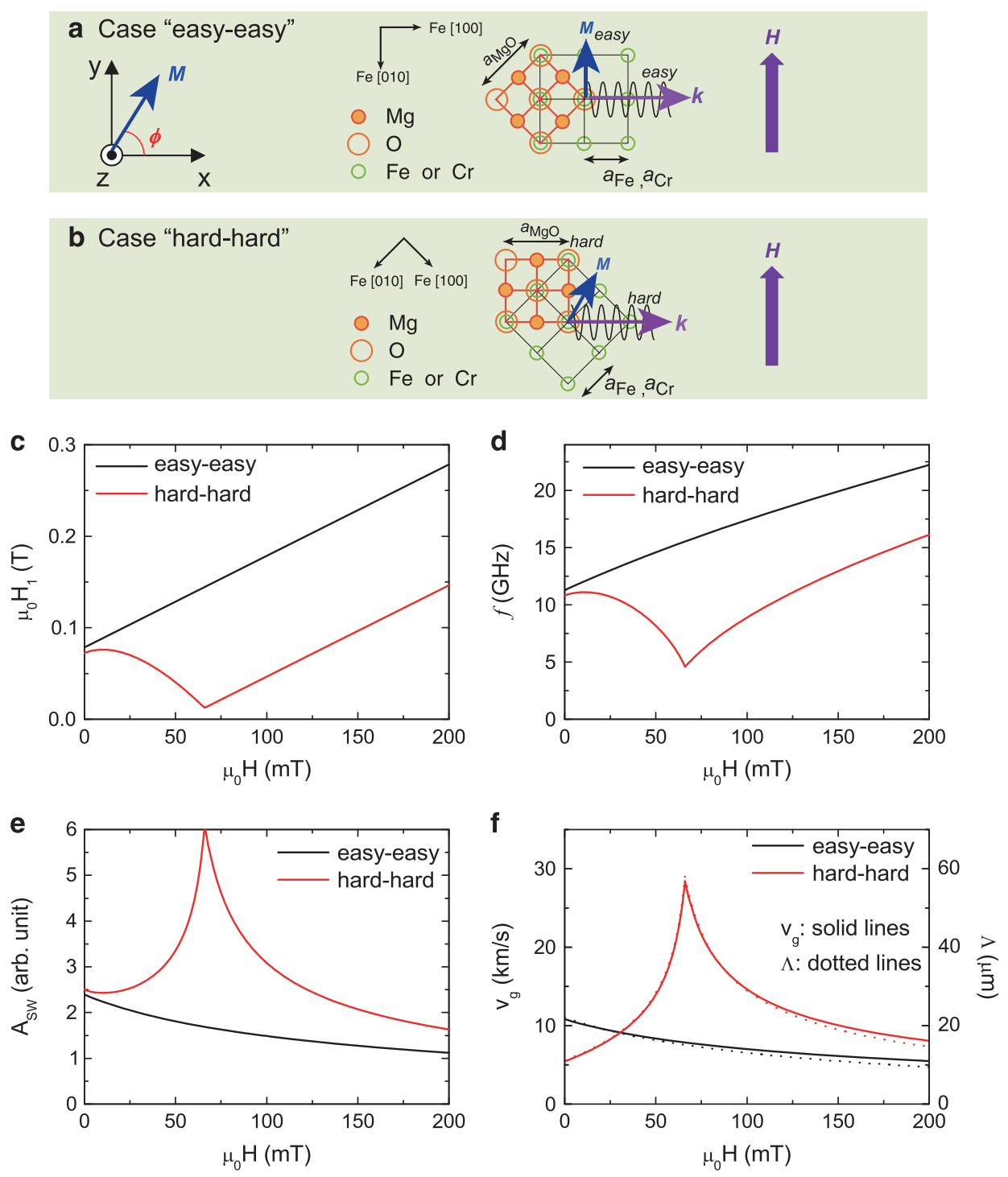

Figure 1 One-dimensional theoretical calculations of spin-wave properties in a cubic anisotropic material. Top view of the planar system of (a) an easy-easy case and (b) a hard-hard case, where the crystallographic orientations are described for an epitaxial Fe layer with a cubic crystalline anisotropy. The long axis of the waveguide (i.e., the direction of wave vector $\mathbf{k}$ ) for the easy-easy device is along the easy axis (Fe [100] direction (a), whereas that for the hard-hard device is along the hard axis (Fe [1]10] direction (b). Computed results were based on spin-wave theories (Equations (1)-(3)). (c) In-plane effective field $H_{1}$ versus external field $H$. (d) Spin-wave frequency $f$ versus $H$. (e) Spin-wave amplitude $A_{\text {sw }}$ versus $H$. (f) Group velocity $v_{g}$ and attenuation length $\Lambda$ versus $H$. Parameters: $M_{\mathrm{S}}=1600 \mathrm{kA} \mathrm{m}^{-1}, \mu_{0} H_{\mathrm{A}}=66 \mathrm{mT}, k=5 \times 10^{5} \mathrm{~m}^{-1}, \gamma_{\mathrm{g}}=1.76 \times 10^{11} \mathrm{~T}^{-1} \mathrm{~s}^{-1}, \alpha=0.0026$ and $d=25 \mathrm{~nm}$.

This localized edge mode at the zero field allows us to significantly improve spin-wave asymmetry using cubic anisotropic materials. It can be compared to the conventional spin-wave non-reciprocity in terms of 'asymmetric propagation of spin wave.' Conventional spin-wave non-reciprocity refers to asymmetric spin-wave amplitude, depending on the spin-wave propagation direction when spin waves are excited by a magnetic field generated by microwave antennas. ${ }^{25-28}$ This amplitude asymmetry results from a non-reciprocal antennaspin-wave coupling, caused by the spatially nonuniform distribution of the antenna field. The asymmetry factor $\left(\equiv A_{\mathrm{SW}}^{\mathrm{k}>0} / A_{\mathrm{SW}}^{\mathrm{k}<0}\right)$ for conventional spin-wave non-reciprocity is $\sim 2 .^{27,28}$ In contrast, the lateral spin-wave asymmetry in cubic anisotropic materials can be very large when the detection antenna is properly designed. Figure $4 \mathrm{e}$ shows that the spin-wave profile with respect to the location of the excitation antenna is highly symmetric at the top and bottom edges. Therefore, by placing a detection antenna at one of the edges (see Figure 4d), one can obtain a very large difference in the spin-wave amplitude between the cases for $\phi=\pi / 4$ and for $\phi=3 \pi / 4$ (the asymmetry factor $\approx 40$ for Figure $4 \mathrm{f}$ ).

To confirm the lateral spin-wave asymmetry, we experimentally measure the spatial distribution of the magnon density by microfocused Brillouin light scattering (BLS) spectroscopy ${ }^{29}$ (see Methods) for the hard-hard device. We inject a radio frequency current at $10.4 \mathrm{GHz}$ in the excitation antenna and measure the BLS spectra at $H=0$ as a function of the distance $x$ from the signal line (Figure 5a). The BLS spectra at an edge (i.e., the bottom edge, $y=115 \mu \mathrm{m}$ ) show a clear dependence on $x$ (Figure 5b). The BLS intensity shows a highly asymmetric distribution with respect to the signal line (Figure $5 \mathrm{c}-\mathrm{f}$ ), in agreement with modeled results. We note (BLS intensity for $x<0$ )/ (BLS intensity for $x>0$ ) $\gg 1$ in Figure 5c, corresponding to giant lateral spin-wave asymmetry. The change in asymmetry depending on the equilibrium magnetization direction is also consistent with 
a

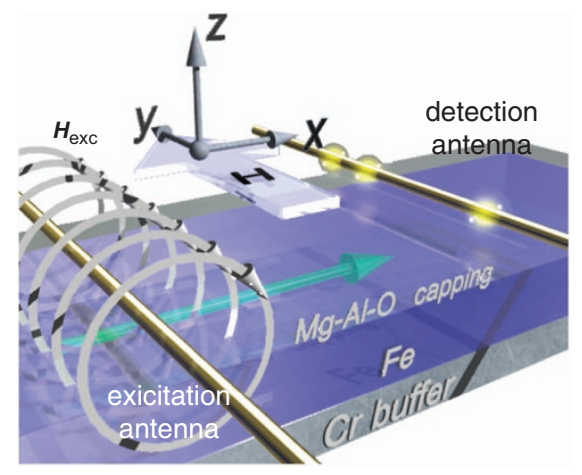

b

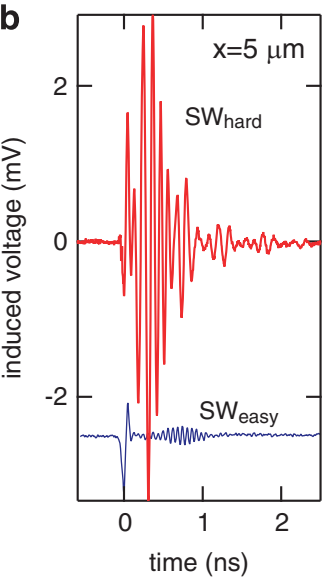

C

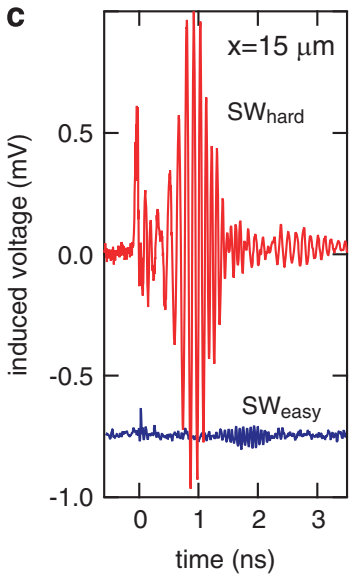

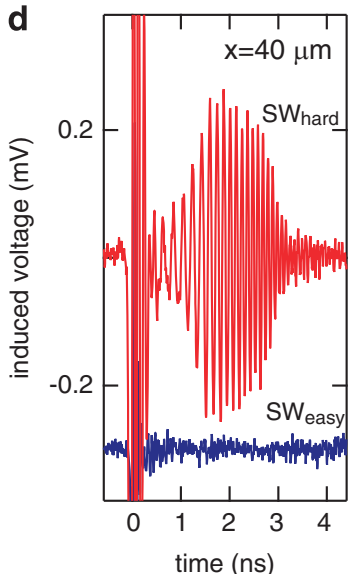

Figure 2 Large-amplitude propagating spin waves in an epitaxial Fe waveguide. (a) Schematic illustration of the time-domain propagating spin-wave spectroscopy. Spin-wave packets at distances of $5 \mu \mathrm{m}$ (b), $15 \mu \mathrm{m}$ (c) and $40 \mu \mathrm{m}$ (d). The external magnetic field is $70 \mathrm{mT}$. At time $t=0$, a pulsed voltage is applied to excite spin waves. In (b-d), the curves are intentionally offset for clarity.

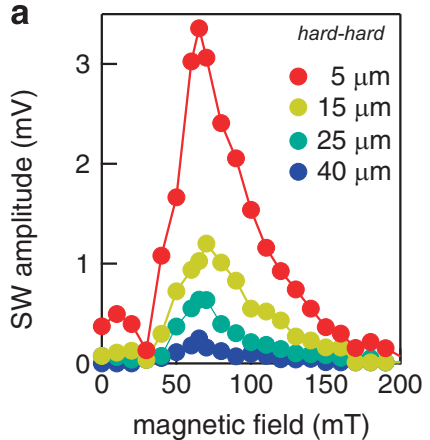

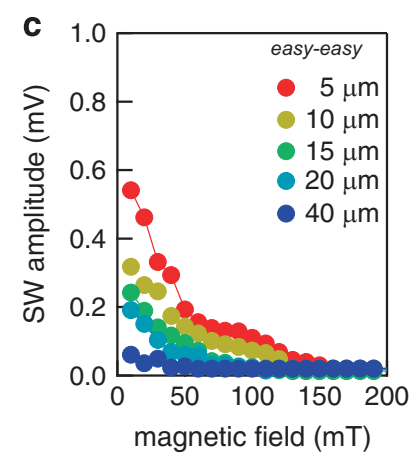
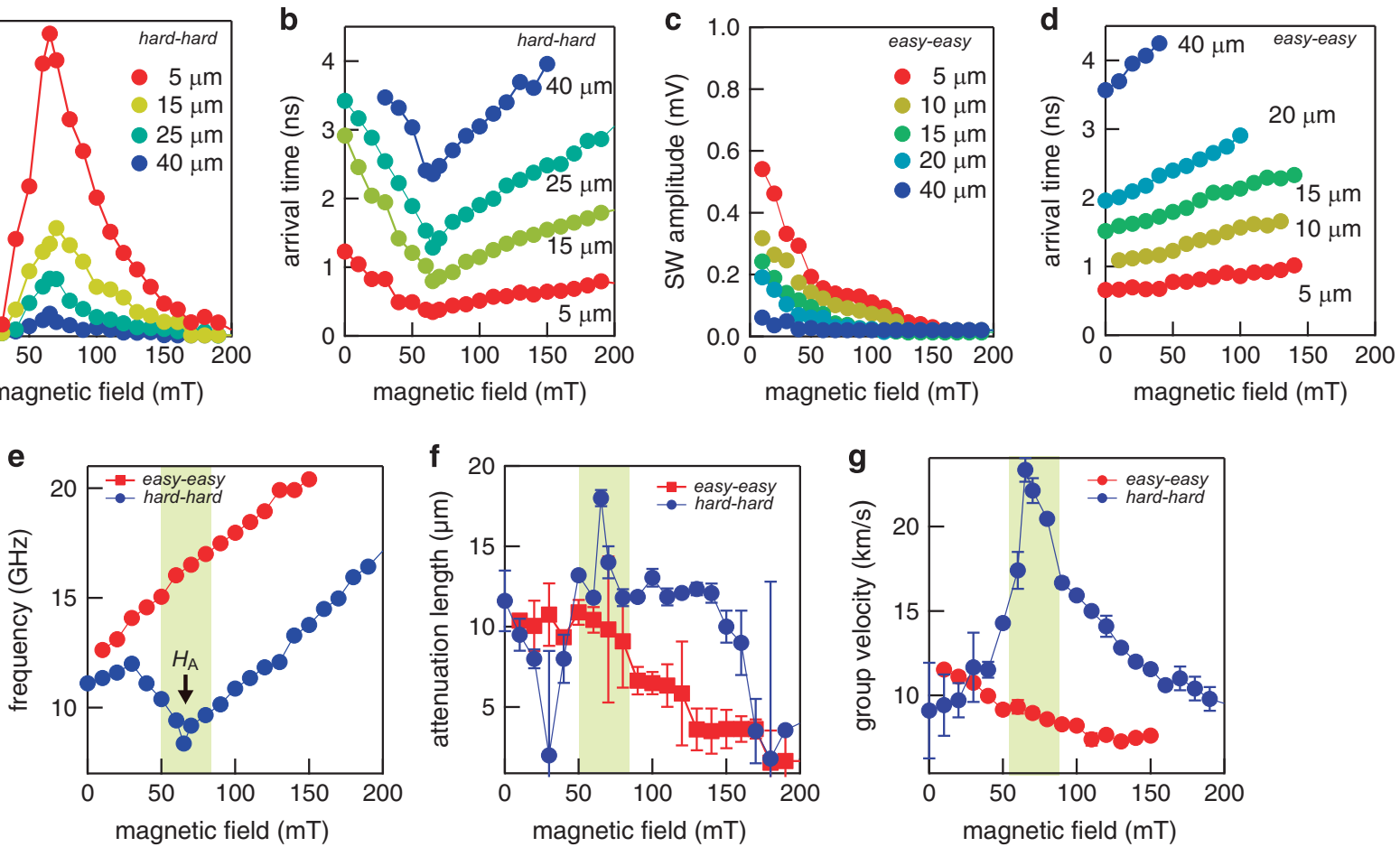

Figure 3 Spin-wave properties in epitaxial Fe waveguide. Magnetic-field dependencies of (a) spin-wave amplitude and (b) arrival time of spin-wave packets for the hard-hard case. (c) Spin-wave amplitude and (d) arrival time of spin-wave packet for the easy-easy case. (e) The spin-wave frequencies deduced by FFT of the time-resolved waveforms. (f) Deduced attenuation length and (g) group velocity as a function of the magnetic field. Where visible, error bars represent single s.d.'s. Otherwise, uncertainties are smaller than the symbols.

modeled results. Thus, these results confirm the formation of a laterally localized edge mode in cubic anisotropic materials, which is tunable by changing the equilibrium magnetization direction.

It is worthwhile to compare the laterally localized edge mode of cubic anisotropy-based spin-wave devices with a gate voltagecontrolled conducting channel of traditional three-terminal field-effect transistors (FETs). A similarity is that in the spin-wave devices, the edge channel is controlled by the equilibrium magnetization direction (which can be set by an external field), whereas in FETs, the conducting channel is controlled by a gate voltage. Therefore, the equilibrium magnetization direction of cubic anisotropy-based spin-wave devices behaves similar to a gate voltage of FETs.
This similarity makes it possible to mimic the functionalities of three-terminal FETs with three-terminal cubic anisotropy-based spin-wave devices by controlling the equilibrium magnetization direction and measuring the induced voltage with an antenna placed at an edge (see Figure $4 \mathrm{~d}$ ). Because of great asymmetry in edge modes, the on/off ratio of spin waves (i.e., on (off) $\equiv$ spin-waveinduced voltage measured at an edge antenna for $\phi=\pi / 4(\phi=3 \pi / 4))$ can be very large.

There is also an important difference. Traditional FETs are volatile because the conducting channel is closed when the gate voltage is turned off. In contrast, the cubic anisotropy-based spin-wave devices are non-volatile because the spin-wave edge channel is maintained by 
a

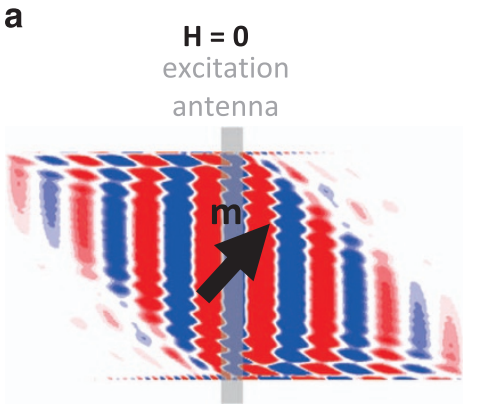

d

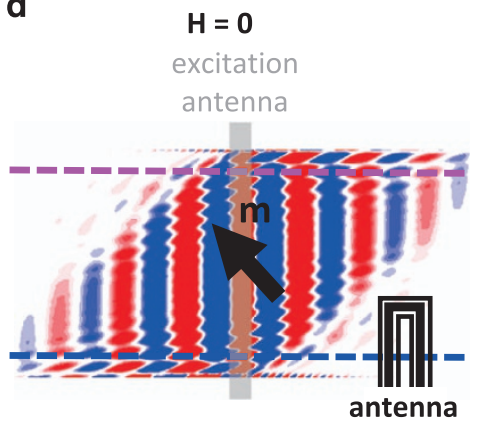

b

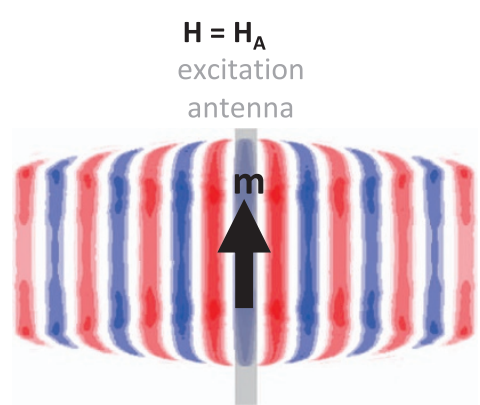

e

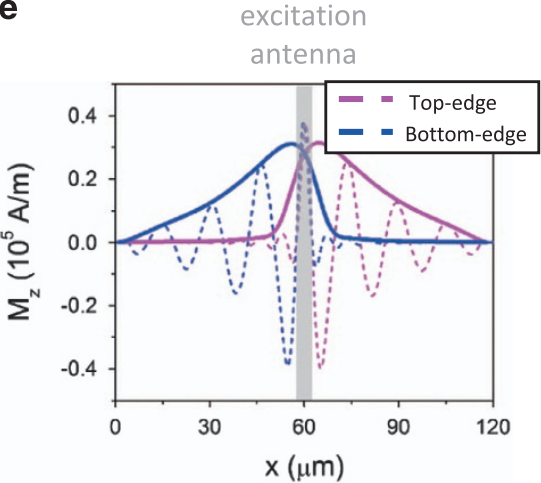

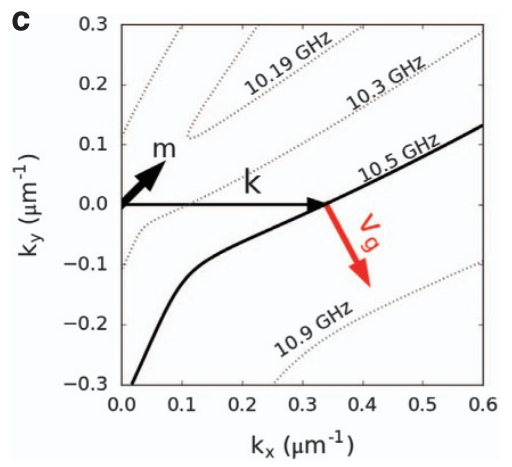

f

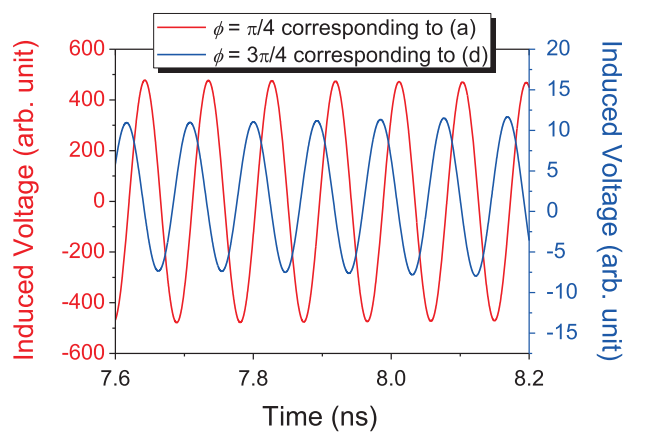

Figure 4 Two-dimensional micromagnetic simulations for laterally localized edge modes. Snapshots of propagating spin waves for (a) $H=0(\phi=\pi / 4$, $f=11.2 \mathrm{GHz})$, (b) $H=H_{\mathrm{A}}(\phi=\pi / 2, f=9.6 \mathrm{GHz})$ and $(\mathbf{d}), H=0(\phi=3 \pi / 4, f=11.2 \mathrm{GHz})$. The direction of magnetization $\mathbf{m}$ is depicted by a black arrow. (c) Contour plot of the spin wave dispersion relation at $H=0(\phi=\pi / 4)$. The contour at the excitation frequency of $10.5 \mathrm{GHz}$ is highlighted. (e) The spin-wave amplitude profile along the top-edge line and bottom-edge line depicted in (d), showing the lateral asymmetry. (f) Induced voltages generated by the laterally asymmetric spin waves (the detection antenna is assumed to be at $40 \mu \mathrm{m}$ from the excitation antenna). The amplitude ratio between two cases is $\sim 40$.

a
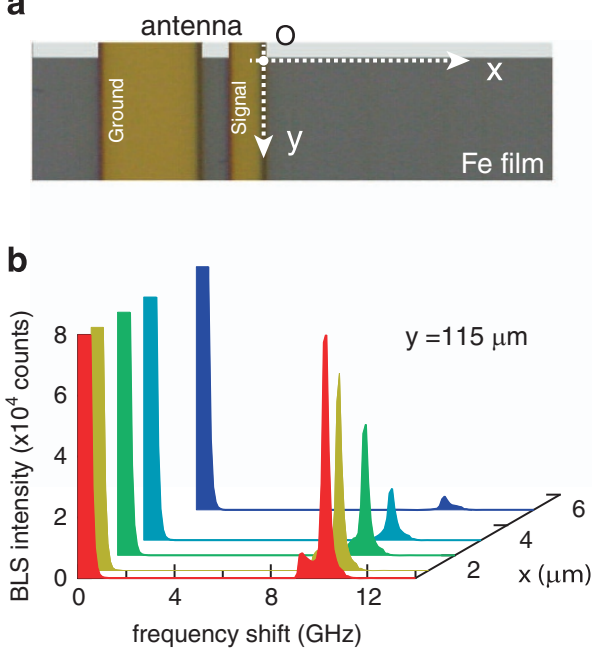

C
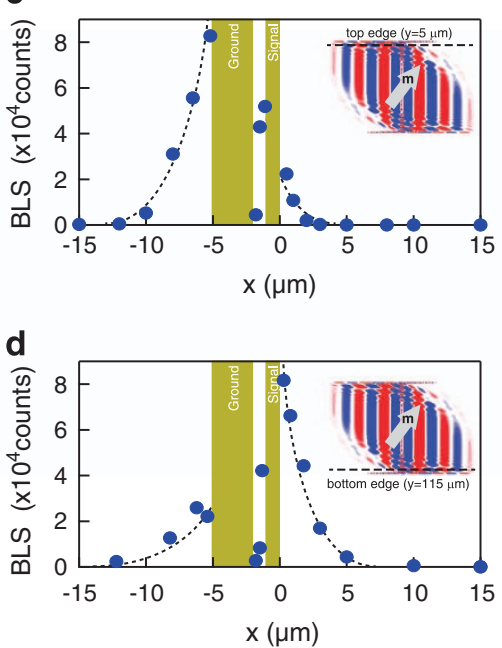

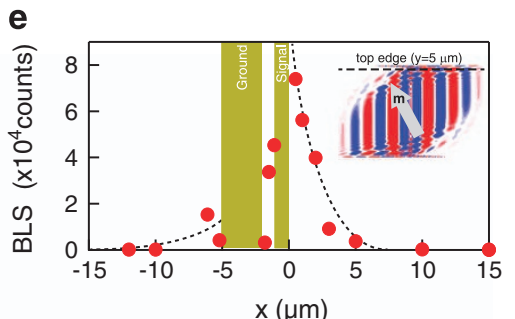

f

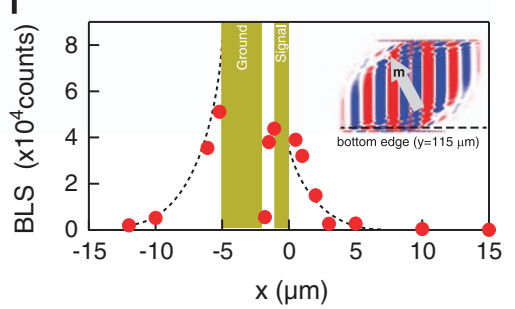

Figure 5 BLS detection of the edge spin-wave mode. Spatial distribution of magnon density in the remanent state $(H=0)$. (a) BLS spectra are obtained at the edges $y=5$ and $115 \mu \mathrm{m}$. (b) Typical BLS spectra at the bottom edge $(y=115 \mu \mathrm{m})$ measured with the frequency window $9<f<12 \mathrm{GHz}, \mathrm{containing}$ a sharp peak centered at an excitation frequency $10.4 \mathrm{GHz}$. Asymmetric distribution of magnon density at (c), the top edge $(y=5 \mu \mathrm{m})$ with $\phi=\pi / 4$, (d) bottom edge $(y=115 \mu \mathrm{m})$ with $\phi=\pi / 4$, (e) top edge $(y=5 \mu \mathrm{m})$ with $\phi=3 \pi / 4$ and (f) bottom edge $(y=115 \mu \mathrm{m})$ with $\phi=3 \pi / 4$. Insets show corresponding micromagnetic simulation results.

the easy-axis anisotropy, even after removing an external field. In this respect, the cubic anisotropy-based spin-wave devices can serve as three-terminal non-volatile logic gates. In Supplementary Note 3, we propose proof-of-principle spin-wave logic gates performing Boolean functions of NOT, PASS, AND, NAND, OR, NOR, XOR and XNOR. We note, however, that the proposed gates have inputs (spin waves with a magnetic field) and outputs (only spin waves) that are different so that they require additional spin-wave-to-current converters, which are definitely detrimental for practical use. One may combine spin-wave logic with spin-transfer torque ${ }^{30}$ or an 
electric-field magnetization switching technique ${ }^{31}$ to remove or at least simplify this additional part.

\section{CONCLUSION}

We have demonstrated that cubic anisotropic materials are a promising candidate for coherent magnonic devices by virtue of largely enhanced spin-wave properties and laterally localized edge modes. The enhanced spin-wave properties will greatly improve the signal-to-noise ratio of magnonic devices. Until now, conventional spin-wave nonreciprocity has been employed to generate a $\pi$ phase-shifted wave $^{27}$ and different spin-wave amplitudes, ${ }^{26-28}$ providing plenty of magnonic functionalities. The lateral asymmetry reported here will add a new functionality: three-terminal non-volatile spin-wave logic function.

\section{METHODS}

\section{Sample preparation}

The devices are deposited using magnetron sputtering with a base pressure of $<7 \times 10^{-7} \mathrm{~Pa}$ on $\mathrm{MgO}(001)$ single-crystal substrates. The high-quality epitaxial $\mathrm{Fe}(001)$ layer is fabricated by the multilayer stack of $\mathrm{Cr}(40) / \mathrm{Fe}(25) / \mathrm{Mg}(0.45) / \mathrm{Mg}_{19} \mathrm{Al}_{81}$ (1.2)/oxidation (thickness in nanometers). ${ }^{22}$ The full-width half-maximum of the rocking curve for the (002)-plane is reached at $0.204^{\circ}$ and the surface roughness is $R_{\mathrm{a}} \leqslant 0.11 \mathrm{~nm}$. The saturation magnetization $M_{\mathrm{S}}=(1.6 \pm 0.1) \times 10^{6} \mathrm{~A} \mathrm{~m}^{-1}$ of epitaxial Fe films is determined by a vibrating sample magnetometer.

\section{Time-domain propagating spin-wave spectroscopy}

Spin waves are excited and detected with a pair of asymmetric coplanar strip transmission lines. The asymmetric coplanar strips are designed to have the $50 \Omega$ characteristic impedance and are patterned by electron beam lithography followed by a lift-off of $\mathrm{Ti}(5 \mathrm{~nm}) / \mathrm{Au}$ $(200 \mathrm{~nm})$. A voltage pulse injected into the excitation asymmetric coplanar strip generates an excitation magnetic field, which in turn excites a spin-wave packet. Propagating spin wave induces a voltage on the detection asymmetric coplanar strip connected to a $20 \mathrm{GHz}$ sampling oscilloscope.

\section{Micromagnetic simulation}

Micromagnetic simulations are performed by numerically solving the Landau-Lifshitz-Gilbert equation, given as $\partial \mathbf{m} / \partial \mathrm{t}=-\gamma_{\mathrm{g}} \mu_{0} \mathbf{m} \times \mathbf{H}_{\mathrm{eff}}+$ $\alpha_{\mathrm{G}} \mathbf{m} \times \partial \mathbf{m} / \partial \mathrm{t}$, where $\mathbf{m}$ is the unit vector along the magnetization, $\mathbf{H}_{\text {eff }}$ is the effective magnetic field including the exchange, magnetostatic and external fields, and $\alpha_{\mathrm{G}}$ is the Gilbert damping. The following parameters are used: the dimensions of the Fe film $=120 \mu \mathrm{m} \times 120 \mu \mathrm{m} \times 25 \mathrm{~nm}$; the dimension of the unit cell $=500 \mathrm{~nm} \times 500 \mathrm{~nm} \times 25 \mathrm{~nm} ; \quad \alpha_{\mathrm{G}}=0.002 ; \quad$ the exchange stiffness constant $A_{\mathrm{ex}}=1.3 \times 10^{-11} \mathrm{~J} \mathrm{~m}^{-1}$; the cubic anisotropy $K_{\mathrm{c}}=5.48 \times 10^{4} \mathrm{~J} \mathrm{~m}^{-13}$; and the saturation magnetization $M_{\mathrm{S}}=1.66 \times 10^{6} \mathrm{~A} \mathrm{~m}^{-1}$.

\section{BLS microscopy}

The spin-wave intensity is detected by BLS microscopy, which is based on the inelastic scattering of photons and magnons. A continuous, single-mode 532-nm solid-state laser is focused on the sample using a high numerical aperture microscope lens. The spatial resolution is $250 \mathrm{~nm}$. The frequency shift of the inelastically scattered light is analyzed using a tandem (six-pass) Fabry-Perot interferometer. The sample position is monitored with a charge-coupled device camera and an active stabilization algorithm, which allows for controlling the sample position with respect to the laser focus with a precision better than $20 \mathrm{~nm}$.

\section{CONFLICT OF INTEREST}

The authors declare no conflict of interest.

\section{ACKNOWLEDGEMENTS}

This work was supported by the Japan Science and Technology Agency, Precursory Research for Embryonic Science and Technology (JST-PRESTO). KS also acknowledges Grants-in-Aid for Scientific Research (25706004, 16K13670, 16H02098) from the Ministry of Education, Culture, Sports, Science and Technology, Japan. KS acknowledges D Chiba and N Ishida for valuable contributions. KJL acknowledges the National Research Foundation of Korea (NRF) grant funded by the Korean government (MSIP) (2015M3D1A1070465, 2017R1A2B2006119) and Korea University Future Research Grant.

Author contributions: KS and K-JL planned the experiment. KS, HS and NS designed and prepared the samples. KS and NS performed the time-domain spin-wave spectroscopy and the BLS microscopy. S-WL, S-HO, RDM and K-JL performed analytical and numerical calculations to verify the effect of cubic magnetic anisotropy on spin-wave properties. KS, S-WL, RDM and K-JL wrote the paper. All authors discussed the results.

1 Serga, A. A., Chumak, A. V. \& Hillebrands, B. YIG magnonics. J. Phys. D 43, 264002 (2010).

2 Kruglyak, V. V., Demokritov, S. O. \& Grundler, D. Magnonics. J. Phys. D 43, 264001 (2010).

3 Lenk, B., Ulrichs, H., Garbs, F. \& Münzenberg, M. The building blocks of magnonics. Phys. Rep. 507, 107-136 (2011).

4 Khitun, A. \& Wang, K. L. Nano scale computational architectures with spin wave bus. Superlattices Microstruct. 38, 184-200 (2005).

5 Schneider, T., Serga, A. A., Leven, B. \& Hillebrands, B. Realization of spin wave logic gates. Appl. Phys. Lett. 92, 022505 (2008).

6 Lee, K.-S. \& Kim, S.-K. Conceptual design of spin wave logic gates based on a Mach-Zehnder-type spin wave interferometer for universal logic functions. J. Appl. Phys. 104, 053909 (2008).

7 Khitun, A., Bao, M. \& Wang, K. L. Magnonic logic circuits. J. Phys. D 43, 264005 (2010).

8 Csaba, G., Papp, A. \& Porod, W. Spin-wave based realization of optical computing primitives. J. Appl. Phys. 115, 17C741 (2014).

9 Sato, N., Sekiguchi, K. \& Nozaki, Y. Electrical demonstration of spin-wave logic operation. Appl. Phys. Express. 6, 063001 (2013).

10 Chumak, A. V., Vasyuchka, V. I., Serga, A. A. \& Hillebrands, B. Magnon spintronics. Nat. Phys. 11, 453-461 (2015).

11 Vogt, K., Fradin, F. Y., Pearson, J. E., Sebastian, T., Bader, S. D., Hillebrands, B., Hoffmann, A. \& Schultheiss, H. Realization of a spin-wave multiplexer. Nat. Commun. 5, 3727 (2014).

12 Vogel, M., Chumak, A. V., Waller, E. H., Langner, T., Vasyuchka, V. I., Hillebrands, B. \& Freymann, G. V. Optically-reconfigurable magnetic materials. Nat. Phys. 11, 487-491 (2015).

13 Haldar, A., Kumar, D. \& Adeyeye, A. O. A reconfigurable waveguide for energy-efficient transmission and local manipulation of information in a nanomagnetic device. Nat. Nanotechnol. 11, 437 (2016).

14 Kwon, J. H., Yoon, J., Deorani, P., Lee, J. M., Sinha, J., Lee, K.-J., Hayashi, M. \& Yang, H. Giant nonreciprocal emission of spin waves in Ta/Py bilayers. Sci. Adv. 2, e1501892 (2016).

15 Khitun, A. Multi-frequency magnonic logic circuits for parallel data processing. J. Appl. Phys. 111, 054307 (2012).

16 Khitun, A. Magnonic holographic devices for special type data processing. J. Appl. Phys. 113, 164503 (2013).

17 Kalinikos, B. A., Kostylev, M. P., Kozhus, N. V. \& Slavin, A. N. The dipole-exchange spin wave spectrum for anisotropic ferromagnetic films with mixed exchange boundary conditions. J. Phys. Condens. Matter 2, 9861-9877 (1990).

18 Damon, R. W. \& Eshbach, J. R. Magnetostatic modes of a ferromagnet slab. J. Phys. Chem. Solids 19, 308-320 (1961).

19 Patton, C. E. Spin-wave instability theory in cubic single crystal magnetic insulators. Phys. Stat. Sol. B 92, 211-220 (1979).

20 Kalinikos, B. A. \& Slavin, A. N. Theory of dipole-exchange spin wave spectrum for ferromagnetic films with mixed exchange boundary conditions. J. Phys. C 19, 7013-7033 (1986)

21 McMichael, R. D. \& Krivosik, P. Classical model of extrinsic ferromagnetic resonance linewidth in ultrathin films. IEEE Trans. Magn. 40, 2-11 (2004). 
22 Sukegawa, H., Miura, Y., Muramoto, S., Mitani, S., Niizeki, T., Ohkubo, T., Abe, K., Shirai, M., Inomata, K. \& Hono, K. Enhanced tunnel magnetoresistance in a spinel oxide barrier with cation-site disorder. Phys. Rev. B 86, 184401 (2012).

23 Covington, M., Crawford, T. M. \& Parker, G. J. Time-resolved measurement of propagating spin waves in ferromagnetic thin films. Phys. Rev. Lett. 89, 237202 (2002).

24 Sekiguchi, K., Yamada, K., Seo, S. M., Lee, K. J., Chiba, D., Kobayashi, K. \& Ono, T. Time-domain measurement of current-induced spin wave dynamics. Phys. Rev. Lett. 108, 017203 (2012).

25 Bailleul, M., Olligs, D. \& Fermon, C. Propagating spin wave spectroscopy in a permalloy film: a quantitative analysis. Appl. Phys. Lett. 83, 972-974 (2003).

26 Demidov, V. E., Kostylev, M. P., Rott, K., Krzysteczko, P., Reiss, G. \& Demokritov, S. 0. Excitation of microwaveguide modes by a stripe antenna. Appl. Phys. Lett. 95, 112509 (2009).

27 Sekiguchi, K., Yamada, K., Seo, S. M., Lee, K. J., Chiba, D., Kobayashi, K. \& Ono, T. Nonreciprocal emission of spin-wave packet in FeNi film. Appl. Phys. Lett. 97, 022508 (2010).

28 Jamali, M., Kwon, J. H., Seo, S. M., Lee, K. J. \& Yang, H. Spin wave nonreciprocity for logic device applications. Sci. Rep. 3, 3160 (2013).

29 Demokritov, S. O., Hillebrands, B. \& Slavin, A. N. Brillouin light scattering studies of confined spin waves: linear and nonlinear confinement. Phys. Rep. 348, 441-489 (2001).
30 Demidov, V. E., Urazhdin, S., Siu, R., Divinskiy, B., Telegin, A. \& Demokritov, S. 0. Excitation of coherent propagating spin waves by pure spin currents. Nat. Commun. 7 , 10446 (2016).

31 Chiba, D., Sawicki, M., Nishitani, Y., Nakatani, Y., Matsukura, F. \& Ohno, H. Magnetization vector manipulation by electric fields. Nature 455, 515-518 (2008)

This work is licensed under a Creative Commons Attribution 4.0 International License. The images or other third party material in this article are included in the article's Creative Commons license, unless indicated otherwise in the credit line; if the material is not included under the Creative Commons license, users will need to obtain permission from the license holder to reproduce the material. To view a copy of this license, visit http:// creativecommons.org/licenses/by/4.0/

(C) The Author(s) 2017

Supplementary Information accompanies the paper on the NPG Asia Materials website (http://www.nature.com/am) 